\title{
COMUNICAÇÃO
}

\section{IDENTIFICAÇÃO DE ISOSPORÍASE EM PACIENTES COM SÍNDROME DE IMUNODEFICIÊNCIA ADQUIRIDA EM SALVADOR, BAHIA}

\author{
Edson D. Moreira Júnior, Nanci Silva, Maria G. M. Barberino, \\ Carlos Brites, Warren D. Johnson Júnior e Roberto Badaró
}

Isospora belli è um coccideo que passou a atrair a atenção da comunidade médica a partir do reconhecimento da sindrome de imunodeficiência adquirida(SIDA) no início da década de $80^{7}$. Embora incomum nos Estados Unidos onde acomete $0,2 \%$ dos pacientes com SIDA (Centers for Disease Control), a isosporiase ocorre mais frequentemente em outras áreas tropicais do mundo, a exemplo do Haiti, onde $15 \%$ dos pacientes com SIDA apresentam a infecção ${ }^{2}$. No Brasil, pelo menos $1 \%$ dos pacientes com SIDA tem diagnóstico associado de isosporiase ${ }^{3} 5$. Neste relato descrevemos 7 casos de diarréia crônica por Isospora belli em pacientes com SIDA em Salvador, Bahia.

Todos os pacientes preenchiam os critérios diagnósticos de SIDA revisados pelo Centers for Disease Control em 19871. O diagnóstico da isosporiase foi estabelecido através de exame direto das fezes após coloração de Ziehl-Neelsen modificada 46 . Resumidamente, a técnica consiste em: a partir de material sedimentado (camada superior de amostra de fezes dissolvida em formalina $10 \%$, após centrifugação por 2 minutos a $1.750 \mathrm{rpm}$ ) um esfregaço é preparado em lâmina de vidro e fixado pelo calor a $70^{\circ}$ por 10 minutos. Se a amostra tem aspecto mucóide, 10 gotas de $\mathrm{KOH}$ a $10 \%$ são acrescentadas ao sedimento e homogeneizado antes de novo esfregaço ser obtido. Após fixação pelo calor a lâmina é recoberta com carbofucsina, aquecida até vaporização e deixada corar por 5 minutos. Caso a lâmina comece a secar, mais corante é adicionado sem novo aquecimento. $\mathrm{O}$ esfregaço é então enxagụado com àgua coîtente, descorado com ácido sulfúrico a $5 \%$ por 30 segundose lavado novamente com água, éscoảndo-se o excęsso. A lâmina é então corada com azul de metileno por um minuto, enxaguada com água e deixada secar em ar ambiente. Cryptosporidium e $I$. belli coram-se ambos

Laboratório de Retrovírus do Hospital Professor Edgar Santos. Universidade Federal da Bahia, Salvador, BA, Brasil. Cornell University Medical College, New York, NY, USA. Órgão financiador: NIH Grant Al 26506-03.

Endereço para correspondência: Dr. Roberto Badaró. R. Joāo das Botas s/n Canela, 40140 Salvador, BA.

Recebido para publicação em 04/10/90. em vermelho brilhante contra um fundo azul, sendo facilmente visualizados ao exame microscópico com aumento de X400 ou X1000. Os oocistos de $I$. belli se distinguem dos de outros coccídios pelo seu tamanho (20 a $30 \mathrm{~mm}$ por 10 a $19 \mathrm{~mm}$ ), forma oval e presença de 2 esporoblastos, em contraste, os oocistos de Cryptosporidium são arredondados com 4 a $6 \mathrm{~mm}$ de diâmetro e apresentam 4 esporoblastos.

Nenhum outro protozoário, bactéria enteropatogenica ou parasito intestinal foi isolado concomitantemente, nestes pacientes, em pelo menos 3 exames de fezes adicionais. Todos pacientes foram tratados com Sulfametoxazol (SMT) + Trimetoprim (TMP) por via oral, na dose de $800 \mathrm{mg} / 160 \mathrm{mg}$, respectivamente, 4 vezes ao dia, durante 10 dias, passando a 2 vezes por dia por 3 semanas, e mantida uma dose ao dia, 3 vezes por semana como profilaxia de recidivas ${ }^{8}$.

As caracteristicas clínicas e epidemiologicas encontradas nos 7 pacientes estão sumarizadas na Tabela 1. Os pacientes apresentavam diarréia aquosa crônica, sem muco ou sangue, com $11 \pm 3$ evacuações/dia e acompanhada de dor abdominal difusa. $\mathrm{O}$ diagnóstico de isosporiase foi obtido no primeiro exame com coloração de Ziehl-Neelsen modificada em todos os casos, a despeito de todos pacientes apresentarem, previamente, pelo menos 2 exames parasitológicos de fezes negativos. Todos pacientes responderam ao tratamento, com resolução da diarréia em 3,5 $\pm 1,7$ dias em média após o início da terapêutica. Nenhum efeito colateral foi observado.

Diarréia é uma manifestação clínica comum em pacientes com SIDA. Na Bahia, de 110 pacientes com SIDA, $76(69 \%)$ apresentavam diarréia e destes, somente $28(37 \%)$ tinham identificação do agente etiógico (ED Moreira Jr: comunicaçāo pessoal, 1990). A não realização de métodos diagnósticos adicionais no estudo da diarréia destes pacientes pode explicar os baixos índices de confirmação etiológica encontrados.

A utilização do método de coloração de ZiehlNeelsen modificado para a pesquisa de coccídeos pouco frequentes na parasitologia médica no nosso meio, Isospora belli e Criptosporidium $s p$, deve ser incluída na rotina de investigação diagnóstica de pacientes com diarréia e SIDA, possibilitando o rápido reconhecimento, através de método simples e 
Comunicação. Moreira Jr ED, Silva N, Barberino MGM, Brites C, Johnson Jr WD, Badaró R. Identificação de isosporiase em pacientes com síndrome de imunodeficiência adquirida em Salvador, Bahia. Revista da Sociedade Brasileira de Medicina Tropical 24: 61-62, jan-mar, 1991

Tabela 1-Sumário das características clínicas e epidemiológicas de 7 pacientes com SIDA e diarréia por I. belli.

\begin{tabular}{lllcc}
\hline Paciente & $\begin{array}{c}\text { Idade (anos) } \\
\text { /sexo }\end{array}$ & $\begin{array}{c}\text { Grupo de } \\
\text { Risco* }\end{array}$ & $\begin{array}{c}\text { Duração da } \\
\text { Diarréia } \\
\text { (meses) }\end{array}$ & $\begin{array}{c}\text { Outras Infecçōes } \\
\text { Oportunistas** }\end{array}$ \\
\hline 1. RCS & $34 / \mathrm{M}$ & Homo & 4 & PPC \\
2. GLS & $39 / \mathrm{M}$ & Homo & 3 & $\mathrm{CE}$ \\
3. ARCM & $24 / \mathrm{F}$ & Het & 3 & TX, CE \\
4. ENA & $36 / \mathrm{M}$ & Bi & 2 & $\mathrm{CO}$ \\
5. XMF & $34 / \mathrm{M}$ & Homo & 3 & $\mathrm{CO}, \mathrm{TB}$ \\
6. ACCO & $35 / \mathrm{M}$ & UDEV & 2 & PPC \\
7. PCD & $31 / \mathrm{M}$ & UDEV & 5 & $\mathrm{CO}$ \\
\hline
\end{tabular}

* Homo = Homossexual, $\mathrm{Bi}=$ Bissexual, Het $=$ Heterossexual, UDEV = Usuário de drogas endovenosas.

** $\mathrm{CE}=$ Candidiase esofágica, $\mathrm{CO}=$ Candidiase oral, $\mathrm{TX}=$ Toxoplasmose em sistema nervoso central, $\mathrm{TB}=$ Tuberculose, PPC $=$ Pneumonia por Pneumocystis carinit.

não invasivo, de infecção tratável e, provavelmente, subestimada em nosso meio.

\section{AGRADECIMENTOS}

Os autores agradecem a Angélica Maria Bastos pela assistência na realização das avaliações microbiológicas e a Jackson Lemos e Josemar de A. Ferreira pela assistência na digitação deste manuscrito.

\section{REFERÊNCIAS BIBLIOGRÁFICAS}

1. Centers for Disease Control: Revision of the CDC surveillance case definition for acquired immunodeficiency syndrome. Morbidity and Mortality Weekly Report 36 (suppl): 3s-15s, 1987.

2. DeHovitz JA, Pape JW, Boncy M, Johnson Jr WD. Clinical manifestations and therapy of Isospora belli infection in patients with the acquired immunodeficiency syndrome. The New England Journal of Medicine 315: 87-90, 1986.

3. Divisão de Doenças Sexualmente Transmissiveis/AIDS do Ministério da Saúde. Boletim Epidemiológico. Ano III n. 11, junho, 1990.

4. Garcia LS, Bruckner DA, Brewer TC, Shimizu RY. Techniques for the recovery and identification of Cryptosporidium oocystis from stool specimens. Journal of Clinical Microbiology 18: 185-190, 1983.

5. Kronfeld M, Sprinz E. Influence of geographic distribution on atypical mycobacteriosis and Isospora belli in Rio Grande do Sul (Brasil). In: Abstract of the Fifth International Conference on AIDS, Montreal th. B.P. 47, 1989.

6. Ma P, Soave R. Three-step stool examination for cryptosporidiosis in 10 homosexual men with protracted watery diarrhea. The Journal of Infectious Disease 147: 824-828, 1983.

7. Ng E, Markell EK, Fleming RL, Fried M. Demonstration of Isospora belli by acid-fast stain in a patient with acquired immunodeficiency syndrome. Journal of Clinical Microbiology 20: 384-386, 1984.

8. Pape JW, Verdier RI, Johnson Jr WD. Treatment and prophylaxis of Isospora belli infection in patients with the acquired immunodeficiency syndrome. The New England Journal of Medicine 320: 1044-1047, 1989. 